\title{
Influência da fisioterapia na reabilitação de pacientes submetidos à drenagem torácica em um hospital de urgência e emergência da amazônia legal
}

\author{
Influence of physical therapy rehabilitation of patients to submit thoracic drainage in \\ hospital of emergency legal amazon
}

Influencia de la fisioterapia en la rehabilitación de pacientes sujetos a drenaje torácico en un hospital de emergencia legal de amazonia

Maycon Pelosato Duarte ${ }^{1 *}$, Ledne Luiz Dalla Rosa1, Erick Marques Pinheiro ${ }^{1}$, Isabela Kerber Alves $^{1}$, Aline Arcari Santos ${ }^{1}$, Állef Diego Bonfim de Andrade ${ }^{1}$, Leiri Bonet ${ }^{1}$, Marcos Vinícius de Azevedo $^{2}$, Welton Alves Diniz ${ }^{3}$.

\section{RESUMO}

Objetivo: Analisar a influência da fisioterapia na reabilitação de pacientes submetidos à drenagem torácica pós-trauma de tórax atendidos em um Hospital de Urgência e Emergência da Amazônia Legal. Métodos: Trata-se de um estudo descritivo, prospectivo, através de uma pesquisa documental de levantamento de dados em um Hospital de Urgência e Emergência da Amazônia Legal, com base na coleta de dados dos prontuários de pacientes que realizaram drenagem torácica pós-trauma de tórax e submetidos à intervenção fisioterapêutica no período de março a setembro de 2018. Resultados: A predominância dos pacientes admitidos com trauma torácico neste estudo é da população adulta jovem, do sexo masculino e vítimas de acidente automobilístico. Notou-se estatisticamente que o tempo de internação hospitalar e o tratamento fisioterapêutico são diretamente proporcionais para uma boa evolução clínica do paciente, pois a reabilitação pulmonar reduziu os índices de complicações pós-drenagem torácica, houve melhora da capacidade pulmonar e tempo de internação reduzido. Conclusão: Dentre os principais objetivos da reabilitação pulmonar notou-se a redução dos índices de complicações pós-drenagem torácica, com melhora da capacidade funcional e da qualidade de vida. Contudo observou-se estatisticamente a correlação entre o tempo de internação e o tratamento fisioterapêutico, que são diretamente proporcionais.

Palavras-chave: Traumatismos torácicos, Serviço hospitalar de fisioterapia, Terapia respiratória,

\begin{abstract}
Objective: To analyze the influence of physiotherapy on the rehabilitation of patients submitted to chest trauma after chest trauma treated at a Legal Amazon Emergency Hospital. Methods: This is a descriptive, prospective study, through a documentary survey of data collection at the Legal Amazon Emergency Hospital, based on data collection from medical records of patients who underwent thoracic drainage after chest trauma and submitted to physical therapy intervention from March to September 2018. Results: The predominance of patients admitted with chest trauma in this study is from the young adult male population and victims of a car accident. It was noted statistically that the length of hospital stay and physiotherapeutic treatment are directly proportional to a good clinical outcome of the patient, as pulmonary rehabilitation reduced the rates of complications after chest drainage, there was an improvement in lung capacity and
\end{abstract}

\footnotetext{
${ }^{1}$ Complexo Hospitalar Regional de Cacoal (COHREC), Cacoal - RO.

*E-mail: maayconpelozato@hotmail.com

${ }^{2}$ Hospital Regional de São Francisco do Guaporé - HRSFG - São Francisco do Guaporé - RO

${ }^{3}$ Instituto de Assistência Médica ao Servidor Público Estadual - IAMSPE - São Paulo - SP
} 
reduced hospitalization time. Conclusion: Among the main objectives of pulmonary rehabilitation was the reduction in the rates of complications after chest drainage, with improvement in functional capacity and quality of life. However, there was a statistically significant correlation between length of stay and physiotherapeutic treatment, which are directly proportional.

Keywords: Thoracic injuries, Physiotherapy hospital service, Respiratory therapy.

\section{RESUMEN}

Objetivo: Analizar la influencia de la fisioterapia en la rehabilitación de pacientes sometidos a traumatismo torácico después de un traumatismo torácico tratado en un Hospital Legal de Emergencias de Amazon. Métodos: Este es un estudio descriptivo, prospectivo, a través de una encuesta documental de recolección de datos en el Hospital Legal de Emergencias de Amazon, basado en la recolección de datos de registros médicos de pacientes que se sometieron a drenaje torácico después de un traumatismo torácico y sometido a intervención de fisioterapia de marzo a septiembre de 2018. Resultados: El predominio de pacientes ingresados con trauma en el pecho en este estudio es de la población de varones adultos jóvenes y víctimas de un accidente automovilístico. Se observó estadísticamente que la duración de la estancia hospitalaria y el tratamiento fisioterapéutico son directamente proporcionales a un buen resultado clínico del paciente, ya que la rehabilitación pulmonar redujo las tasas de complicaciones después del drenaje torácico, hubo una mejora en la capacidad pulmonar y el tiempo de hospitalización. Conclusión: Entre los objetivos principales de la rehabilitación pulmonar se encontraba la reducción de las tasas de complicaciones después del drenaje torácico, con una mejora en la capacidad funcional y la calidad de vida. Sin embargo, hubo una correlación estadísticamente significativa entre la duración de la estadía y el tratamiento fisioterapéutico, que son directamente proporcionales.

Palabras clave: Lesiones torácicas, Servicio hospitalario, Terapia respiratoria.

\section{INTRODUÇÃO}

O serviço de urgência e emergência é a principal porta de entrada de um hospital para o paciente que apresenta alterações importantes do ponto de vista biofísico e psíquico, necessitando de um atendimento imediato, pois apresenta risco iminente de morte (PICCOLI A, et al., 2013; CAMERRO A, et al., 2015).

O trauma é caracterizado por lesões que envolvem diversas regiões anatômicas, gera um grande impacto na sociedade, e isso tem sido a principal causa de óbito e internação hospitalar. O traumatismo torácico tem se destacado em virtude de fatores vinculados a urbanização, e pelo aumento da violência urbana. Constitui uma das mais importantes causas de morbimortalidade na população adulta jovem, pois suas vítimas, quando sobrevivem, apresentam um tempo prolongado de hospitalização (PADOVANI C, et al., 2017; OMS, 2009).

$\mathrm{Na}$ última década, o trauma ocupava o terceiro lugar em causas de mortes no Brasil, os dados mostraram 150 mil óbitos e 450 mil pacientes com sequelas por ano (BRASIL, 2010). Segundo números do Ministério da Saúde referentes ao Sistema Único de Saúde, foram registrados 86.563 internações decorrentes do trauma até o mês de dezembro de 2016 (DATASUS, 2016).

Diversas causas, tais como traumas, atos cirúrgicos e doenças pleurais podem provocar acúmulo de gás ou líquido na cavidade pleural, de forma a alterar o sistema pressórico, determinando condições patológicas como o colapso pulmonar e insuficiência respiratória de intensidade variável (CIPRIANO FG, DESSOTE LU, 2011).

Nessas situações clínicas, principalmente nos casos de trauma torácico, a toracotomia com drenagem tubular em sistema fechado está indicada. $O$ objetivo da drenagem torácica é remover o acúmulo de líquido ou ar alojado na cavidade pleural, possibilitando a reexpansão pulmonar e o restabelecimento da pressão subatmosférica do espaço para evitar complicações (NISHIDA G, et al., 2011). 
O sistema de drenagem torácica fechada em selo d'água é uma técnica na qual é inserido um tubo na linha média axilar lateral do corpo entre as costelas. O liquido é drenado por um sistema coletor de drenagem composto de frasco coletor, tubo de drenagem e selo d'agua que impede a entrada de ar atmosférico na cavidade torácica (UTTER GH, 2013). Este procedimento tem sido descrito como o principal método para o tratamento imediato do hemotórax e pneumotórax traumáticos. Do mesmo modo, os drenos torácicos permitem o controle de hemorragias intratorácicas, a avaliação quantitativa e qualitativa do conteúdo drenado e a redução do risco de infecções, visto que o espaço pleural pode tornar-se um importante meio de cultura (LIGHT RW, 2011).

As principais limitações respiratórias observadas em pacientes acometidos por trauma torácico são: fraturas de arcos costais, pneumotórax, hemotórax, derrame pleural, hidrotórax, deslocamento de dreno, enfisema subcutâneo e fístula bilateral (NÓBREGA KCC, et al., 2012). Tais limitações apresentam alterações significantes na mecânica ventilatória dos pacientes que são submetidos à drenagem torácica, que envolve a elasticidade e a complacência pulmonar, gerando prejuízo na capacidade vital, no volume corrente e na capacidade residual funcional (OLIVEIRA JB, et al., 2005).

Alguns autores sugerem que os pacientes com dreno de tórax devem ser incluídos em programas de reabilitação respiratória precocemente. A atuação do fisioterapeuta se dá após a conduta emergencial de drenagem (ULUBAY G, et al., 2015; VALENZA G, et al., 2014). A fisioterapia é a ciência destinada a promover a recuperação e preservação da funcionalidade através do movimento humano, por meio das suas intervenções, enquadra-se com ênfase na assistência aos pacientes e de gestão na equipe multiprofissional com atuação em diversos campos na área da saúde (ÉRIKO E, et al., 2012).

Ainda nesse contexto Machado MGR (2008) descreveu que a fisioterapia respiratória participa desde a prevenção até o tratamento das doenças pulmonares, empregando-se técnicas e recursos terapêuticos que objetiva a restauração de um padrão ventilatório funcional com o intuito de minimizar os gastos energéticos durante a inspiração e a expiração. Desta forma, favorece o indivíduo a realizar diversas atividades cotidianas sem ocasionar grandes desordens e repercussões negativas a homeostase do seu organismo.

A reabilitação pulmonar por meio de fisioterapia respiratória utiliza técnicas e recursos tais como: respiração diafragmática, sustentação máxima de inspiração, padrão respiratório em exercícios ativos associado com os membros superiores e inferiores, incentivadores respiratórios e pressão positiva através da ventilação não invasiva (MARCHI E, MUSSI R, 2010), consequentemente a reexpansão pulmonar propicia grande mobilidade das estruturas que compõem a caixa torácica, proporcionando o retorno gradativo da mecânica respiratória, através do aumento da expansibilidade torácica nos diâmetros anteroposterior e lateral, reduz as complicações das vítimas de trauma torácico (ABREU EMS, et al., 2015).

Sendo assim, o objetivo da pesquisa foi analisar a influência da fisioterapia na reabilitação de pacientes submetidos à drenagem torácica pós-trauma de tórax atendidos em um Hospital de Urgência e Emergência da Amazônia Legal.

\section{MÉTODOS}

O presente estudo foi elaborado por meio de uma pesquisa transversal de caráter descritivo e prospectivo, com abordagem quantitativa através de uma pesquisa documental de levantamento de dados estatísticos coletados em um hospital de urgência e emergência da Amazônia Legal com base na coleta de dados dos prontuários de pacientes que realizaram drenagem torácica pós-trauma de tórax no período de março a setembro de 2018. A pesquisa iniciou logo após a aprovação no Comitê de Ética Pesquisa sob o parecer $n^{\circ}=3.201 .087$.

Os critérios de inclusão foram: pacientes adultos de ambos os sexos; maiores de 18 anos; submetidos à drenagem torácica; admitidos no hospital entre março a setembro de 2018 e aqueles que receberam intervenção fisioterapêutica durante o período de internação. Já os critérios de exclusão foram: pacientes que não foram submetidos à drenagem torácica; pacientes com menos de 24 horas de internação no hospital; crianças; gestantes e prontuários sem registros. 
Os dados foram prospectivamente coletados a partir da análise de prontuários no período de março a setembro de 2018, com a utilização de formulário com 16 questões pré-estabelecidas elaboradas pelos próprios pesquisadores, baseados nas seguintes variáveis: idade, gênero, mecanismo do trauma, local de internação, tempo de permanência do dreno, tempo de internação hospitalar, presença de complicações e intervenção fisioterapêutica realizada.

\section{RESULTADOS}

Foram selecionados 33 prontuários dos pacientes para análise. Após a primeira triagem, 12 prontuários, que atenderam aos critérios de inclusão, foram selecionados para a tabulação dos dados, a Tabela 1 demonstra os valores dessas variáveis.

Tabela 1 - Variáveis de gênero, idade, diagnóstico, mecanismo do trauma, tempo de internação hospitalar, tempo de permanência do dreno torácico, complicações, conduta fisioterapêutica e quantidade de atendimentos fisioterapêuticos.

\begin{tabular}{|c|c|}
\hline Variáveis & Valores expressos em média e porcentagem \\
\hline Idade (anos) & 33,83 \\
\hline \multicolumn{2}{|l|}{ Gênero (\%) } \\
\hline Masculino & $92(\mathrm{n}=11)$ \\
\hline Feminino & $8(n=1)$ \\
\hline \multicolumn{2}{|l|}{ Diagnóstico (\%) } \\
\hline Politrauma & $58(n=7)$ \\
\hline Ferimento por arma de fogo (FAF) & $33(n=4)$ \\
\hline Ferimento por arma branca (FAB) & $9(n=1)$ \\
\hline \multicolumn{2}{|l|}{ Mecanismo do trauma (\%) } \\
\hline Acidente motociclístico & $58(n=7)$ \\
\hline Agressão física (FAB e FAF) & $42(n=5)$ \\
\hline \multicolumn{2}{|l|}{ Complicações (\%) } \\
\hline Hemotórax & $33(n=4)$ \\
\hline Pneumotórax & $33(n=4)$ \\
\hline Hemopneumotórax & $25(n=3)$ \\
\hline Derrame pleural & $9(n=1)$ \\
\hline Tempo de internação hospitalar (dias) & 7,8 \\
\hline Tempo de dreno torácico (dias) & 7 \\
\hline \multicolumn{2}{|l|}{ Conduta fisioterapêutica (quantidade) } \\
\hline Incentivadores respiratórios & 18 \\
\hline Deambulação & 14 \\
\hline Tosse manualmente assistida & 14 \\
\hline Manobras de reexpansão pulmonar & 13 \\
\hline Cinesioterapia & 13 \\
\hline Mobilização articular & 12 \\
\hline Exercícios respiratórios & 9 \\
\hline Posicionamento & 7 \\
\hline Média de atendimento fisioterapêutico & 6,5 \\
\hline
\end{tabular}

Fonte: Pelosato MD, et al. (2020)

A avaliação primária é muito importante, visto que, quando analisados em relação às doenças pulmonares, estes dados podem influenciar no sucesso da recuperação pulmonar em indivíduos com dreno de tórax. Por isso, se faz necessário conhecer a história clínica prévia, idade, gênero, diagnóstico, mecanismo do trauma e as possíveis complicações. 
No presente estudo, estes dados foram analisados e a predominância dos pacientes admitidos com trauma torácico no hospital é da população adulta jovem com média de idade de 33,83 anos, do sexo masculino e vítimas de acidente automobilístico (Tabela 1). Estes achados corroboram com o estudo de Costa CA, et al. (2005) que relataram incidência de trauma torácico em população jovem, urbana, predominantemente masculina e relacionada à violência interpessoal.

O mecanismo de trauma predominante consistiu em contusão torácica por acidente motociclístico (58\% dos casos), seguido por ferimento penetrante por projétil de arma de fogo e arma branca ( $42 \%$ dos casos) (Tabela 1). Oikonomou A, Prassopoulos $P$ (2011) mostraram que o trauma torácico é o responsável por alta morbimortalidade, cerca de $25 \%$ das mortes são relacionadas a este tipo trauma, atrás apenas do traumatismo cranioencefálico. Os mecanismos de trauma são classificados em penetrante ou contuso, sendo este último responsável por $90 \%$ dos casos, na qual a maioria é por acidente motociclístico.

As complicações respiratórias que tiveram maior incidência foram hemotórax e o pneumotórax (33\%), respectivamente, seguido de hemopneumotórax (25\%) e derrame pleural (9\%), em ambos os gêneros (Tabela 1). A principal e mais comum indicação para a drenagem de tórax é o hemopneumotórax com prevalência de $52,3 \%$, seguida por $23,4 \%$ nos casos de hemotórax, $20,7 \%$ nos casos de pneumotórax e também por casos de derrame pleural (MORALES CH, 2014).

Na maioria dos casos a ocorrência dessas complicações causa distúrbio ventilatório restritivo, diminuição da expansibilidade pulmonar dentro da caixa torácica e alteração na mecânica pulmonar, acarretando em redução do volume pulmonar (OLIVEIRA BM, MEJIA DPM, 2012).

A fisioterapia respiratória dispõe de uma variedade de técnicas e recursos que são capazes de influenciar a mecânica pulmonar com exercícios que objetivam modificar o grau de participação dos músculos respiratórios com a finalidade de aumentar a ventilação pulmonar. O volume pulmonar pode ser aumentado em decorrência da técnica de reexpansão pulmonar que provoca um aumento no gradiente de pressão transpulmonar, ou seja, quanto maior o gradiente de pressão transpulmonar, maior será a expansão das unidades alveolares (GIACOMAZZI CM, et al., 2012).

De acordo com o estudo de Jablonskin S, et al. (2014), a média de tempo de utilização do dreno torácico foi de 4 dias e tempo de internação hospitalar foi de 7 dias ao comparar técnicas cirúrgicas na colocação do dreno de tórax. No presente estudo, foi observado um tempo médio de utilização do dreno torácico de 7 dias e de internação hospitalar de 7,8 dias e a média de atendimento fisioterapêutico nesses pacientes foi de 6,5 dias.

Uma das estratégias clínicas que pode ser adotada para acelerar a remoção do dreno torácico é a fisioterapia respiratória com técnicas de reexpansão pulmonar, sendo este um dos critérios para remoção do dreno. Na pesquisa de Valenza G, et al. (2014) foi observado que os pacientes que realizaram fisioterapia durante o tratamento de drenagem torácica apresentaram uma média inferior de tempo de internação de 27 dias quando comparados em relação ao grupo controle que não realizou acompanhamento fisioterapêutico e apresentou 39 dias.

\section{DISCUSSÃO}

Dentre as várias intervenções fisioterapêuticas encontrada nos dados dos pacientes submetidos à drenagem torácica pós-trauma de tórax no hospital podemos destacar o uso dos incentivadores respiratórios, sendo a conduta mais utilizada nesses pacientes. As demais condutas utilizadas foram: a deambulação, a tosse manualmente assistida, as manobras de reexpansão pulmonar, a cinesioterapia, a mobilização articular, os exercícios respiratórios e o posicionamento desses pacientes (Tabela 1).

A quantidade total e a média dos atendimentos fisioterapêuticos estão apresentadas na Tabela 1. Sendo que, na maioria dos casos, a intervenção fisioterapêutica consistia na associação da fisioterapia respiratória em conjunto com a fisioterapia motora, onde foi observado melhor evolução do quadro clínico dos pacientes. 
Ulubay G, et al. (2015) e Valenza G, et al. (2014) recomendaram que os pacientes com dreno de tórax devem ser incluídos em programas de reabilitação respiratória precocemente, uma vez que a atuação do fisioterapeuta se dá após a conduta emergencial de drenagem torácica.

Diversos autores sugeriram que a fisioterapia respiratória restabelece precocemente a função pulmonar, após drenagem torácica, reduz a incidência de complicações pulmonares, favorece a função circulatória, diminui a incidência de fenômenos tromboembólicos, evita o processo de descondicionamento físico sofrido durante a hospitalização, remoção precoce do dreno torácico e redução do tempo de internação hospitalar (VALENZA-DEMET G, et al., 2014; SILVEIRA AP, et al., 2011).

O acompanhamento fisioterapêutico precoce pós-drenagem torácica promove aumento da saturação periférica de oxigênio, redução da ocorrência de atelectasias, assim como a redução dos custos hospitalares (WALTRICK T, CUNEN SK, 2012).

Pena FF, et al. (2011) mostraram a atuação da fisioterapia respiratória logo após a colocação do dreno torácico, através de incentivadores respiratórios, ventilação por pressão positiva e orientações gerais quanto ao quadro clínico. Entretanto, nesse estudo, os pacientes que realizaram intervenção fisioterapêutica após a colocação do dreno de tórax não obtiveram um desfecho satisfatório quanto melhora no tempo de internação, porém, houve melhora na ventilação e complacência pulmonar e na oxigenação arterial.

De acordo com a necessidade e a condição clínica do paciente, o atendimento fisioterapêutico poderá ser feito de duas a três vezes por dia, a importância da fisioterapia é de, com suas técnicas, promover uma recuperação muito rápida, contribuindo para a redução significativa do tempo de internação (HADDAD ER, COSTA LCD, 2011).

O campo de atuação da fisioterapia respiratória vem se destacando no âmbito hospitalar por apresentar resultados benéficos para a recuperação dos pacientes críticos e, principalmente, atuando na prevenção de complicações clínicas e redução do tempo de internação hospitalar, com consequente redução de custos do tratamento. Desta forma, com a abordagem fisioterapêutica, os pacientes que são submetidos à drenagem torácica podem apresentar diminuição dos sintomas e melhora da capacidade funcional e qualidade de vida (CUNHAL C, et al., 2009).

Com a realização de fisioterapia respiratória no ambiente hospitalar, os pacientes submetidos à drenagem torácica podem apresentar diminuição da dispneia e melhora da capacidade funcional e qualidade de vida (COSTA LRN, 2015). Esses achados corroboram com Cohen M, Sahn SA (2012) ao mostrarem que o tratamento fisioterapêutico é recomendado para evitar o acúmulo de líquido pleural, reduzir a probabilidade de atelectasias, evitar acúmulo de secreções, diminuir a respiração paradoxal e melhorar a mobilidade torácica.

Outros benefícios das técnicas fisioterapêuticas incluem prevenir complicações pulmonares através da melhora da ventilação pulmonar e da efetividade do mecanismo da tosse. Além disso, permite manter a mobilidade da caixa torácica e correção de padrões respiratórios ineficientes por meio da coordenação dos músculos ventilatórios, promovendo resistência à fadiga respiratória e melhora da capacidade funcional geral dos pacientes (DUARTE J, HELFSTEIN TT, 2011).

Martins FC, Virtuoso LA (2009) destacaram que os objetivos da fisioterapia respiratória em pacientes com trauma torácico são prevenir e tratar as complicações decorrentes das alterações funcionais, a fim de melhorar as capacidades e volumes pulmonares para obter uma adequada oxigenação e ventilação.

A reabilitação pulmonar por meio de fisioterapia respiratória utiliza técnicas e recursos tais como: respiração diafragmática, sustentação máxima de inspiração, padrão respiratório em exercícios ativos associado com os membros superiores e inferiores, incentivadores respiratórios e pressão positiva através da ventilação não invasiva (MARCHI E, MUSSI R, 2010).

Existem alguns recursos utilizados para a realização da fisioterapia respiratória para reexpansão pulmonar tais como as manobras manuais, a pressão positiva contínua nas vias aéreas, a pressão positiva em dois níveis pressóricos, a pressão positiva expiratória e o incentivador respiratório, que são recursos seguros e de fácil aplicação (ANJOS APG, 2016). 
O fisioterapeuta pode realizar técnicas reexpansivas com pressão positiva intermitente, para aliviar a sintomatologia destes pacientes. A utilização de pressão positiva intermitente deve ser instituída somente após a drenagem para reexpansão de unidades alveolares que permaneçam colapsadas. A ventilação mecânica invasiva deve-se preconizar 0 uso de pressão expiratória positiva final na reexpansão de atelectasias, assim como manutenção de ventilação alveolar adequada (NÓBREGA KCC, et al., 2012; SARMENTO GJV, 2005).

Os exercícios respiratórios são técnicas manuais de reexpansão pulmonar que são frequentemente empregadas na prática clínica. Estes podem alterar o padrão respiratório, que muitas vezes, encontra-se em ritmo paradoxal e, em conjunto com o movimento toracoabdominal são capazes de priorizar um compartimento da caixa torácica em relação ao outro e, desta forma modificam o grau de contração dos músculos respiratórios (VIEIRA DSR, et al., 2014).

Sarmento GJV (2005) descreveu as mesmas técnicas utilizadas em drenagem pleural, adicionando as técnicas posicionamento adequado, preferência em decúbito contralateral, favorecendo uma maior absorção de líquido por linfático. O fisioterapeuta deve orientar aqueles pacientes que estão conscientes e colaborativos para o cuidado com o circuito de drenagem durante a movimentação no leito, com isso prevenir quadros álgicos e possíveis acidentes com os drenos.

Já a retirada do paciente do leito através da sedestação em poltrona, do ortostatismo e a deambulação são condutas fundamentais para a reabilitação do paciente com trauma torácico, devendo ser estimulada de forma precoce e sempre que liberada pela equipe médica (PADOVANI C, et al., 2017).

Guizilini S, et al. (2010) relataram que as técnicas de reexpansão pulmonar podem ser realizadas através de manobras manuais, orientadas pelo fisioterapeuta e/ou com a utilização de aparelhos. Dentre as técnicas fisioterapêuticas que são utilizadas na fase precoce do tratamento após a drenagem torácica, destacam-se as manobras e recursos para reexpansão pulmonar, o trabalho de padrões respiratórios com estimulação do diafragma em diferentes posicionamentos, as orientações posturais, assim como a sedestação e deambulação precoce (COSTA LRN, 2015).

Natalio MA, et al. (2010) utilizaram um protocolo de reabilitação pulmonar em indivíduos com derrame pleural, baseado nas manobras de higiene brônquica e reexpansão pulmonar por pressão negativa, seu resultado foi eficaz na melhora do quadro radiológico e na ausculta pulmonar. Souza AG, et al. (2009) utilizaram algumas técnicas de reexpansão pulmonar após a drenagem do tórax como a sustentação máxima inspirada, os soluços inspiratórios, os padrões respiratórios associados com membros superiores e o uso de incentivadores respiratórios, com o objetivo de melhorar a troca gasosa, a ventilação pulmonar e evitar maiores complicações do tórax acometido.

Diante disso, os autores concluíram que a fisioterapia respiratória foi útil para a melhora da capacidade pulmonar total e, que quando iniciada de forma precoce e efetiva, a mesma promove uma rápida recuperação do paciente, diminuindo assim, o tempo de internação hospitalar. É importante ressaltar que para alcançar os resultados positivos é primordial um amplo estudo do quadro clínico apresentado por esses pacientes, além de uma criteriosa avaliação das condições clínicas desse indivíduo e de um tratamento precoce e condizente com suas necessidades físicas por meio da intervenção fisioterapêutica.

Desta forma, a fisioterapia respiratória é parte essencial na reabilitação de novas complicações respiratórias nos pacientes com trauma torácico, principalmente para prevenir e tratar vários aspectos das desordens respiratórias decorrentes dos traumatismos torácicos, tais como obstrução do fluxo aéreo, retenção de secreção, alterações da função ventilatória, dispneia, melhora no desempenho de exercícios físicos e da qualidade de vida (GOSSELINK R, 2006).

\section{CONCLUSÃO}

A reabilitação através da fisioterapia tornou-se fundamental como parte complementar no tratamento aos pacientes submetidos à drenagem torácica decorrente do trauma torácico. Dentre os principais objetivos da reabilitação pulmonar notou-se a redução dos índices de complicações pós-drenagem torácica, com 
melhora da capacidade funcional e da qualidade de vida. Contudo, observou-se estatisticamente a correlação entre o tempo de internação e o tratamento fisioterapêutico, que são diretamente proporcionais. Desta forma, observa-se que com a fisioterapia o paciente com trauma torácico apresenta uma melhor evolução com tempo de internação reduzido e diminuição de complicações relacionadas ao quadro clínico. Novos estudos poderão complementar os dados obtidos nesta pesquisa e promover embasamento para estratégias de prevenção, em todos os níveis de atenção, dos traumatismos torácicos, assim como a avaliação dos recursos fisioterapêutico empregados em seu tratamento pós-drenagem torácica e outros procedimentos cirúrgicos.

\section{REFERÊNCIAS}

1. ABREU SEM, et al. Impacto de um protocolo de cuidados a pacientes com trauma torácico drenado. Rev. Col. Bras. Cir. 2015, 42 (4): 231-237.

2. ANJOS APG. Fisioterapia respiratória com pressão positiva intermitente: uma análise comparativa em relação ao tratamento convencional na remoção do dreno de tórax. Dissertação de mestrado da Universidade Estadual de Campinas - UNICAMP. Campinas, SP: [s.n]. 2016.

3. BRASIL. Ministério da Saúde. Relatório Saúde Brasil 2009. Uma análise da situação de saúde e da agenda nacional e internacional de prioridades em saúde. Brasília, 2010.

4. CAMERRO A, et. al. Perfil do atendimento de serviços de urgência e emergência. Revista Fafibe On-Line. 2015, Bebedouro/SP, 8(1): 515-524.

5. CIPRIANO FG, DESSOTE LU. Drenagem pleural. Revista FMRP-USP. Medicina (Ribeirão Preto). 2011,44 (1): $70-$ 8.

6. COHEN M, SAHN SA. Resolution of pleural effusions. Chest, 2012, p 60-62.

7. COSTA CA, et al. Traumatismos de veia cava inferior. Rev. Col. Bras. Cir. 2005, vol.32 nํ5, Rio de Janeiro Sept./Oct.

8. COSTA LRN, et al. Impacto da Fisioterapia na Reabilitação de Pacientes Submetidos à Drenagem Torácica em um Hospital de Referência em Urgência e Emergência da Região Metropolitana de Belém-PA. Prática Hospitalar. 2015, Ano XVII, № 102, Nov-Dez.

9. CUNHAL C, et al. Técnicas reexpansivas no derrame pleural: uma revisão de literatura. Rio de Janeiro. Cadernos UniFOA, 2009.

10. DATASUS: Tecnologia da Informação a Serviço do SUS. Brasília: DATASUS; 2016. Disponível em: http://tabnet.datasus.gov.br/cgi/tabcgi.exe?sih/cnv/fruf.def.

11. DUARTE J, HELFSTEIN TT. Estudo comparativo das técnicas de cinesioterapia respiratória convencional e associado às diagonais de membros superiores na reexpansão torácica em indivíduos adultos jovens. J Health Sci Inst. 2011, 29 (3): 198-201.

12. ÉRIKO E, et al. Fisioterapia em pacientes críticos adultos: recomendações do Departamento de Fisioterapia da Associação de Medicina Intensiva Brasileira. Rev Bras Ter Intensiva. 2012; 24 (1): 6-22.

13. GIACOMAZZI CM, et al. A dor como contribuinte do prejuízo na função pulmonar. Rev Bras Cir Cardiovasc. 2012;21(4):386-92.

14. GOSSELINK R. Physical therapy in adults with respiratory disorders: where are we? Rev Bras Fisioter. 2006; $4(10): 361-72$.

15. GUIZILINI S, et al. Efeitos do local de inserção do dreno pleural na função pulmonar no pós-operatório de cirurgia de revascularização do miocárdio. Rev Bras Cir Cardiovasc. 2010;19(1):47-54

16. HADDAD ER, COSTA LCD. Abordagens fisioterapêuticas para remoção de secreções das vias aéreas em recémnascidos: relato de casos. Centro Universitário de Araraquara (Uniara), 2011.

17. JABLONSKIN S, et al. Efficacy assessment of the drainage with permanent airflow measurement in the treatment of pneumothorax with air leak. Thorac Cardiovasc Surg. 2014, 62 (06): 509-515.

18. LIGHT RW. Pleural controversy: Optimal chest tube size for drainage. Respirol. 2011, 16 (1): 244-248.

19. MACHADO MGR. Bases da Fisioterapia Respiratória: Terapia Intensiva e Reabilitação. 1ª ed. Rio de Janeiro: Guanabara Koogan, 2008.

20. MARCHI E, MUSSI R. Derrame Pleural Parapnêumonico e Empiema, Jornal de Pneumologia, $2010 ;$ no9 v.32.

21. MARTINS FC, VIRTUOSO LA. Perfil dos pacientes atendidos pela Fisioterapia no setor de emergência e urgência do Hospital Geral do Grajaú. Anais $11^{\circ}$ Congresso de Iniciação Científica, 5ª mostra de pós-graduação. São Paulo. 2009.

22. MORALES $\mathrm{CH}$, et al. Negative pleural suction in thoracic trauma patients: A randomized controlled trial. $J$ trauma Acute Care Surg. 2014, 77: 251-255.

23. NATALIO MA, et al. Tratamento Fisioterapêutico no Derrame pleural: estudo de caso. Revista Digital Buenos Aires, 2010; ano 14, n.142.

24. NISHIDA G, et al. Cuidados com o sistema de drenagem torácica em adultos internados no Hospital Universitário Regional de Maringá, Estado do Paraná, Brasil. Acta Scientiarum. Health Sciences. 2011, v. 33, n. 2, p. 173-179.

25. NÓBREGA KCC, et al. Intervenção fisioterapêutica em casos de pacientes admitidos por trauma torácico: um estudo retrospectivo. Estação Científica (UNIFAP), 2012, v. 2, n. 1, p. 43-54, jan./jun. 
26. OIKONOMOU A, PRASSOPOULOS P. CT imaging of blunt chest trauma. Insights Imaging. 2011, 2: $281-295$.

27. OLIVEIRA BM, MEJIA DPM. Intervenção fisioterapêutica no derrame pleural: revisão de literatura. [periódico da internet]. 2012.

28. OLIVEIRA JB, et al. A influência da drenagem torácica intercostal fechada com selo d'água na respiração e voz, em sujeitos hospitalizados com doenças pleuro pulmonares. Rev. Fisioterapia Brasil. v. 6, n. 2, mar./abr. 2005.

29. PADOVANI C, et al. Fisioterapia nos pacientes politraumatizados graves: modelo de assistência terapêutica. Acta Fisiatr. 2017; 24 (1):33-39

30. PENA FF, et. al. Crianças com derrame pleural: caracterização e fisioterapia. Rev terapia manual, $2011 ; 9$ (46): 744-748.

31. PICCOLI A, et al. Indicações para Inserção do Profissional Fisioterapeuta em uma Unidade de Emergência. Revista ASSOBRAFIR Ciência. 2013, v. 4, n. 1, p. 33-41.

32. SARMENTO GJV. Fisioterapia respiratória no paciente crítico: Rotinas clínicas. São Paulo: Manole, 2005.

33. SILVEIRA AP, et al. Comparação do uso da pressão positiva com a fisioterapia convencional e incentivadores respiratórios após cirurgia cardíaca: revisão de literatura. Revista FMRP-USP. Medicina (Ribeirão Preto), 2011,44 (4): 338-46.

34. SOUZA AG, et al. Atuação da fisioterapia em crianças com derrame pleural: revisão de literatura. Fassam, 2009.

35. ULUBAY G, et al. Postoperative pulmonary complications in living-liver donors: a retrospective analysis of 188 patients. Exp Clin Transplant. 2015; 13 Suppl 1:340-5.

36. UTTER GH, The rate of pleural fluid drainage as a criterion for the timing of chest tube removal: theoretical and practical considerations. Ann Thoracic Surg. 2013; 96: 2262-7.

37. VALENZA G, et al. The effects of a physiotherapy programme on patients with a pleural effusion: a randomized controlled trial. Clin Rehabil. 2014, 28 (11): 1087-95.

38. VIEIRA DSR, et al. Breathing exercises; influence on breathing pattern and thoracoabdominal motion in healthy subjects. Braz J PhysTher. 2014, 18 (6): 544-552.

39. WALTRICK T, CUNEN SK. Benefícios das Técnicas de fisioterapia respiratória em pacientes com dreno pleural vitimizados por trauma torácico. Rev. Inspirar. 2012, 4 (4):9-15.

40. WORLD HEALTH ORGANIZATION. Guidelines for trauma quality improvement programmes. Geneva: WHO, 2009. 